\title{
Addendum
}

\section{A Green Chemistry Method for the Regeneration of Carbonyl Compounds from Oximes by Using Cupric Chloride Dihydrate as a Recoverable Promoter for Hydrolysis}

Na Quan, Xiao-Xin Shi, * Liang-Deng Nie, Jing Dong, Rui-Heng Zhu Synlett 2011, 1028.

In the recent publication an important reference which was reported by Professor Ram of the Indian Institute of Technology, Delhi (Latika Singh, Ram N. Ram Synth. Commun. 1993, 23, 3139) was missing. Professor Ram's paper discusses an elegant method for the deoximation of oximes with copper(II) chloride dehydrate under reaction conditions very similar to those reported by the authors. The authors regret and feel upset for missing it during literature search. 